\title{
Statistical Analysis of Low-latitude Pi2 Pulsations Observed at Bohyun Station in Korea
}

\author{
Chae-Woo Jun ${ }^{1 \dagger}$, Khan-Huk Kim¹, Hyuck-Jin Kwon ${ }^{1}$, Dong-Hun Lee ${ }^{1}$, Ensang Lee ${ }^{1}$, \\ Young-Deuk Park², and Junga Hwang ${ }^{2}$ \\ ${ }^{1}$ School of Space Research, Kyung Hee University, Yongin 446-701 Korea \\ ${ }^{2}$ Space Science Research Center, Korea Astronomy and Space Science Institute, Daejeon 305-348, Korea
}

We statistically investigated the properties of low-latitude Pi2 pulsations using Bohyun $\left(\mathrm{BOH}, \mathrm{Mlat}=29.8^{\circ}, \mathrm{L}=1.35\right)$ ground magnetometer data in 2008. For this 1-year interval, $582 \mathrm{Pi} 2$ events were identified when $\mathrm{BOH}$ was in the nightside from 1800 to 0600 local times. We found the following Pi2 characteristics. (1) The occurrence distribution of Pi2s is relatively constant in local times. (2) The Pi2 frequency varies in local times. That is, Pi2 pulsations in postmidnight sector had higher frequency than in premidnight sector. (3) Pi2 power in premidnight sector is stronger than in postmidnight sector. (4) Pi2 frequency has positive correlation with solar wind speed and AE index. (5) Pi2 power has not a clear correlation with solar wind parameters. This indicates that Pi2 power is not controlled by external sources. (6) It is found that the most probable-time between Pi2 onsets is $\Delta \mathrm{t} \sim 37.5 \mathrm{~min}$ : This is interpreted to be the period between Pi2 pulsations when they occur cyclically. We suggest that $\Delta \mathrm{t} \sim 37.5 \mathrm{~min}$ is the occurrence rate of reconnection of open field lines in the tail lobe.

Keywords: geomagnetic field, low-latitude Pi2 pulsation, geomagnetic activity.

\section{INTRODUCTION}

Pi2 pulsations have been used as an important indicator for the onset of magnetospheric substorm because the pulsations occur almost simultaneously at the onset of substorm (Saito et al. 1976). The Pi2 pulsations are hydromagnetic waves with the period of $40 \sim 150 \mathrm{~s}$, and they are observed on the ground in the nightside at large areas ranging from high latitudes to low latitudes, and also observed in the nightside of the near-Earth space together with the occurrence of substorm.

Takahashi et al. (1995) studied the spatial characteristics of Pi2 pulsations generated from the Earth's magnetosphere using the data from the Active Magnetospheric Particle Tracer Explorers Charge Composition Explorer (AMPTE CCE) satellite and low-latitude Kakioka ground magnetometers. Takahashi et al. (1995) examined the coherence and radial phase variation between the Pi2 pulsations observed in space and at the low-latitude ground stations. When the CCE satellite is located in the inner magnetosphere $(\mathrm{L}<$ 4) on the nightside, the Pi2 pulsations observed in space was coherent with the low-latitude Pi2 pulsations, and there was no phase difference. Based on this observation, the inner magnetosphere Pi2 pulsations can be explained by the plasmaspheric resonance waves trapped in the plasmasphere.

The plasmaspheric resonance is the wave energy trapped in the plasmasphere, and its existence was directly identified using the electric and magnetic field data of Pi2 pulsations observed from the inner magnetosphere satellite (Takahashi \& Liou 2004, Kim et al. 2010, Kwon et al. 2012).

Nose (2010) investigated whether the low-latitude Pi2 pulsations are caused by the cavity mode resonance or by the bursty bulk flow. The result of this study suggested that (c) This is an open Access article distributed under the terms of the Creative Commons Attribution Non-Commercial License (http:// creativecommons.org/licenses/by-nc/3.0/) which premits unrestricted non-commercial use, distribution, and reproduction in any medium, provided the original work is properly cited.
Received Nov 19, 2012 Revised Jan 22, 2013 Accepted Jan 30, 2013 †Corresponding Author

E-mail: farmshrimp@hotmail.com

Tel: +82-31-201-2690, Fax: +82-31-202-1885 
the sum of Kp index over the entire day is related with the size of plasmasphere, and that the ion mass or F10.7 index of plasma sheet can be substituted with the mass density of plasmasphere. Through the result, the period of Pi2 pulsations was shown to be proportional to the size and mass density of plasmasphere, and based on this, it was concluded that the occurrence of low-latitude Pi2 pulsations is closely associated with the plasmaspheric cavity mode resonance.

Cheng et al. (2002) studied the two low-latitude Pi2 pulsations that occur consecutively using the Sino Magnetic Array at Low Latitudes (SMALL) magnetic field observation network. Cheng et al. (2002) observed that the dominant frequency of consecutive Pi2 pulsations is $10 \sim 20 \mathrm{mHz}$, and that the frequency of the first $\mathrm{Pi} 2$ pulsation increases as the $\mathrm{Kp}$ index increases. In the result of interplanetary magnetic field observation using the Advanced Composition Explorer (ACE) and Wind satellites, the two consecutive $\mathrm{Pi} 2$ pulsations showed different characteristics. The first $\mathrm{Pi} 2$ pulsation occurred when the interplanetary magnetic field points south, and the second Pi2 pulsation occurred as the interplanetary magnetic field turns to north. The first Pi2 pulsation is known to be associated with the magnetic field reconnection phenomenon as the flux of magnetic field originating from the dayside is accumulated at the Earth's magnetotail. And the second Pi2 pulsation is explained by the reconnection of open magnetic lines of force which are located at the magnetotail lobe region. However, the time interval between the two Pi2 pulsations was not mentioned.

This study analyzed the local time distribution of lowlatitude Pi2 pulsations observed at Bohyun ground magnetic field station using the data of the period when the Earth's magnetosphere is not in extremely quiet condition $(\mathrm{Kp}>2)$, investigated the correlation between the solar wind condition and the Pi2 pulsation frequency and pulsation strength, and examined the time interval between the consecutive Pi2 pulsations. In Section 2, the data used in this study and the Pi2 pulsation selection method were described, and in Section 3, the statistical characteristics of $\mathrm{Pi} 2$ pulsations were mentioned. The observation results were discussed in Section 4, and this study was summarized in Section 5.

\section{DATA AND PI2 PULSATION DETECTION}

The BOH magnetic field data during 1 year for 2008 was used to investigate the low-latitude $\mathrm{Pi} 2$ pulsations. The longitude of Bohyun ground station is $128.9^{\circ} \mathrm{E}$, the magnetic $\mathrm{L}$ shell is 1.35 , and the local time is $\mathrm{UT}+9$. In the case of magnetic field data, the 1-second data was averaged for 5 seconds, which was then used for the statistical analysis. The AE index and Kp index were used to understand the perturbation of geomagnetic field. Regarding the AE index and Kp index, the data was obtained from the World Data Center for Geomagnetism, Kyoto (http://wdc.kugi.kyoto-u. ac.jp/index.html). The interplanetary magnetic field data and the magnetic field strength, speed, density and dynamic pressure data for solar wind collected from the ACE satellite were utilized. As for the solar wind data from the ACE satellite, the 1-minute data which was time shifted to the bow shock nose that is provided by the National Aeronautics and Space Administration (NASA) was used (http:// omniweb.gsfc.nasa.gov/).

In this study, the Pi2 pulsations were established with the use of following conditions. The data obtained when the Bohyun magnetic field station is located on the nightside (18 06 local time) was used, and the time series analysis was performed by removing the data values averaged for 5 minutes from the raw data. The Pi2 power value was calculated by integrating the frequency domain $(5 \sim 25 \mathrm{mHz})$ of Pi2 pulsations in 3 minute interval using the Fourier transform method of time series data. With the automated procedure (Takahashi et al. 1995), the Pi2 pulsations were sampled so that the calculated $\mathrm{H}$-component power $\left(\mathrm{P}_{\mathrm{H}}\right)$ value is more than $0.02\left(10^{-1.7}\right) \mathrm{nT}^{2}$ and the maximum $\mathrm{P}_{\mathrm{H}}$ value is 10 times higher than the preceding 9 minute values and the following 17 minute values. Lastly, only the Pi2 pulsations which occurred when the Kp index value excluding the quiet condition is higher than 2 were sampled. And through this method, total $582 \mathrm{Pi} 2$ pulsations were selected.

\section{CASE ANALYSIS}

Fig. 1 shows the AE index, Bohyun magnetic field $\mathrm{H}$-component, and the Mt. Bohyun H-component and Pi2 power which removed the 5-minute average values, that were observed at 04 06 LT on November 9, 2008. Fig. 1d clearly shows the 3 independent Pi2 powers which exceed the threshold value for 2 hours. The vertical dashed line indicates the time that the maximum $\mathrm{Pi} 2$ power value is observed which was obtained by the Pi2 pulsation sampling method. At 04:16 LT, the Pi2 pulsation was observed overlapping with the positive bay. The positive bay is generally observed in mid-low latitudes along with the occurrence of substorm, and this means that the Bohyun ground station is located within the substorm current wedge (Clauer \& McPherron 1974). The variation of $\mathrm{AE}$ index when the substorm occurs is generally about $100 \mathrm{nT}$. However, in this event, the AE index increased about $30 \mathrm{nT}$, and this 

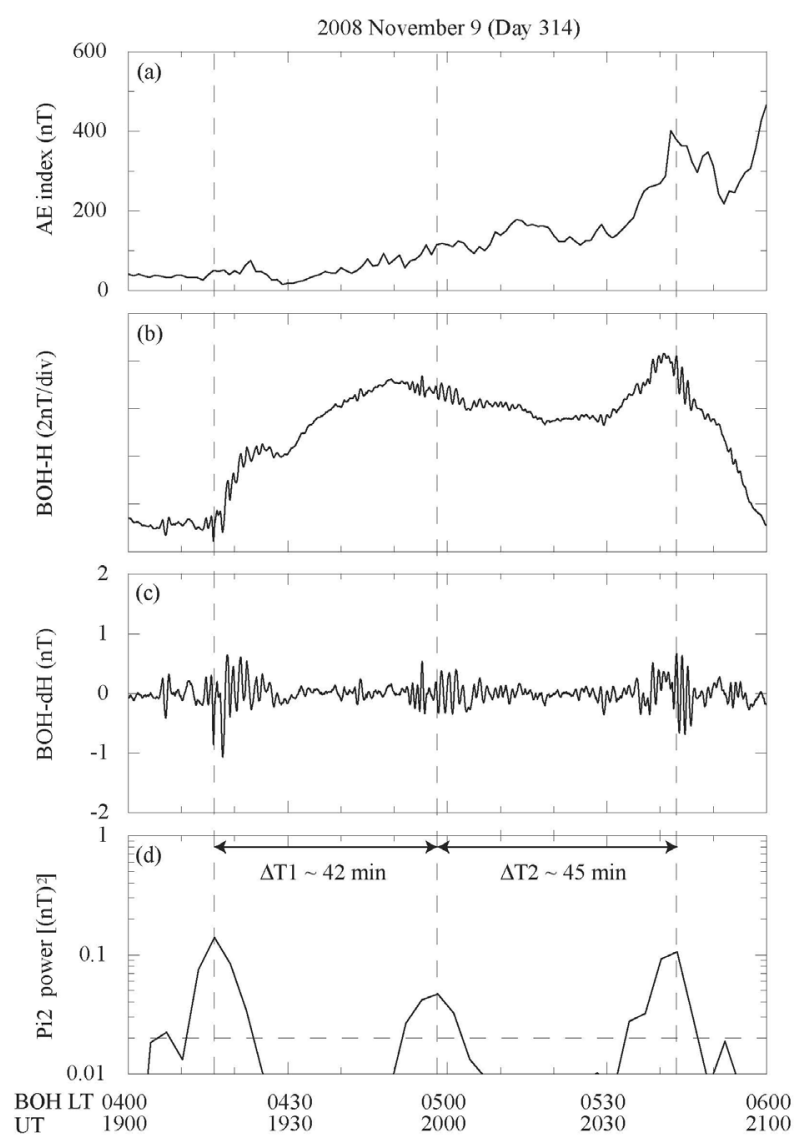

Fig. 1. The ground station data from 0400 to 0600 LT on 9 November 2008. The vertical dashed lines indicate Pi2 pulsation onset time. (a) The AE index, (b) The $\mathrm{H}$-component at $\mathrm{BOH}$ ground station, (c) Magnetometer data from $\mathrm{BOH}$, filtered in the high-pass filter, (d) The Pi2 pulsation power calculated by BOH-dH.

could be classified as a pseudo-substorm (Koskinen et al. 1993, Ohtani et al. 1993, Nakamura et al. 1994). For the second Pi2 pulsation at 04:58 LT, the positive bay was not observed and there was no abrupt variation of AE index. For the third Pi2 pulsation at 05:43 LT, the positive bay and the increase of AE index (about $250 \mathrm{nT}$ ) were observed in the Bohyun H-component. The time intervals for the 3 events were $\Delta \mathrm{T} 1=42$ minutes and $\Delta \mathrm{T} 2=45$ minutes, respectively.

The speed, density and dynamic pressure for solar wind and the interplanetary magnetic field Bz-component observed by the ACE satellite were time shifted to the bow shock nose of Earth's magnetosphere, which are shown in Fig. 2. The vertical dashed line indicates the time that the maximum Pi2 power value is observed. The interplanetary magnetic field Bz-component pointed south for almost 2 hours excluding the time that the first Pi2 pulsation occurred, and the average value was about 2.5 $\mathrm{nT}$. The average solar wind speed was about $450 \mathrm{~km} / \mathrm{s}$, the average density was $2.5 \mathrm{~cm}^{-3}$, and the average solar wind

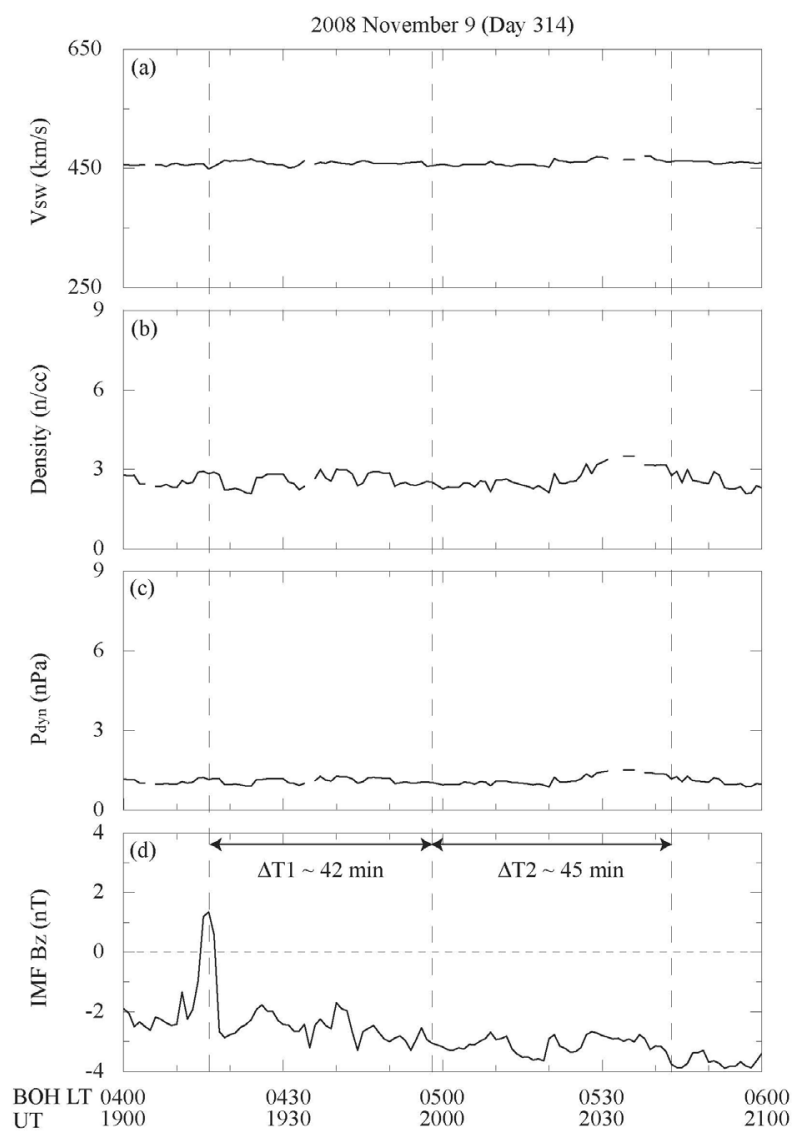

Fig. 2. The ACE satellite data from 0400 to 0600 LT on 9 November 2008, time-shifted on bow shock front. The format is the same as in Fig. 1. (a) Solar wind speed, (b) Solar wind density, (c) Solar wind dynamic pressure, (d) IMF Bz.

dynamic pressure was about $1 \mathrm{nPa}$. And there were no large variations during 2 hours.

\section{STATISTICAL ANALYSIS}

Fig. 3 shows the event number distribution based on local time. In Fig. 3, the histogram represents the number of Pi2 pulsation occurrence in an interval of 1 hour for the time that Pi2 pulsations occurred, which were observed during 2008 when the Bohyun magnetic field station was located on the nightside. As for the nightside, the Pi2 pulsations were evenly distributed from 19:00 to 04:00 LT, and relatively small number of pulsations were observed from 04:00 to 06:00 LT.

Fig. 4 is the Pi2 frequency distribution data based on local time. In Fig. 4, the gray dot represents the data for each Pi2 pulsation frequency observed during 2008 when the Bohyun magnetic field station was located on the nightside. 


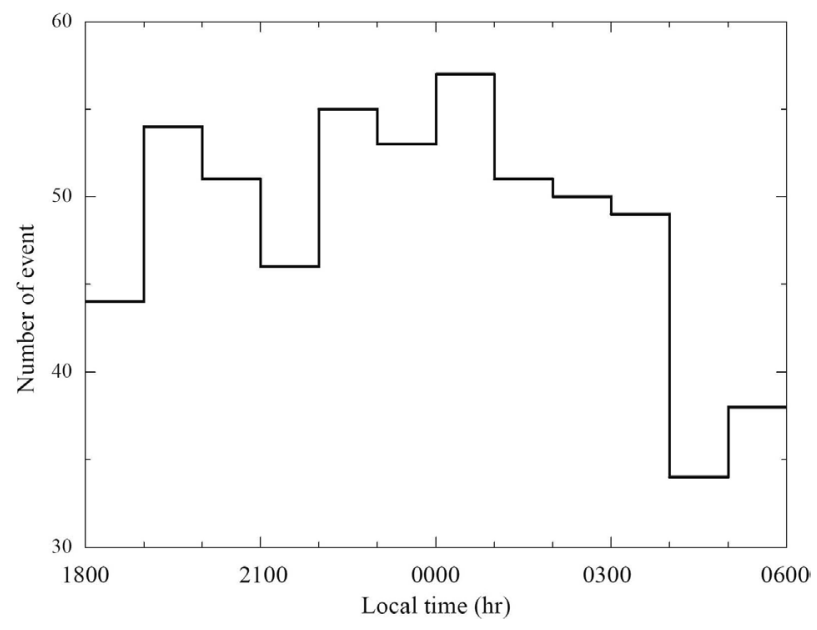

Fig. 3. Occurrence histogram of Pi2 pulsations in LT.

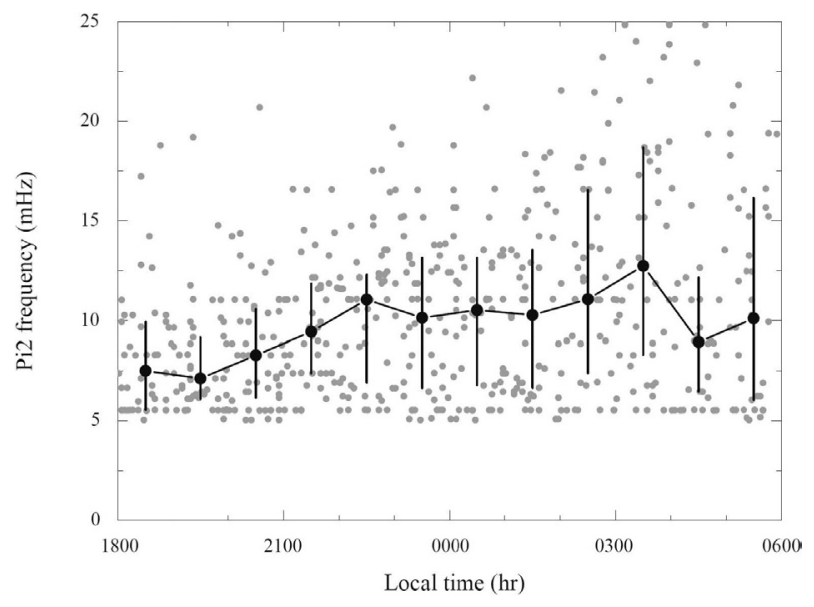

Fig. 4. Frequency of Pi2 pulsation at Bohyun ground station. The small gray dots are individual samples, the heavy black dots are medians in hourly LT bins, and the vertical error bars connect the upper and lower quartiles.

The black dot represents the median value of Pi2 pulsation frequency obtained within the 1-hour interval, and the black vertical line connects the low quartile and upper quartile. The Pi2 pulsation frequency was obtained by the following method. The Pi2 pulsation frequency was sampled so that the Pi2 pulsation power obtained from $5 \sim 25 \mathrm{mHz}$ during the time that the Pi2 pulsations occurred has the maximum value. In this data, the $\mathrm{Pi} 2$ pulsation frequency mostly showed increasing trend from 18:00 to 04:00 LT. This result is consistent with the result of recent $\mathrm{Pi} 2$ pulsation study which used the Thermal Emission Imaging System (THEMIS) satellite data and Bohyun data (Kwon et al. 2012).

Fig. 5 is the Pi2 power data based on local time. According to the variation of median value, the Pi2 power has the

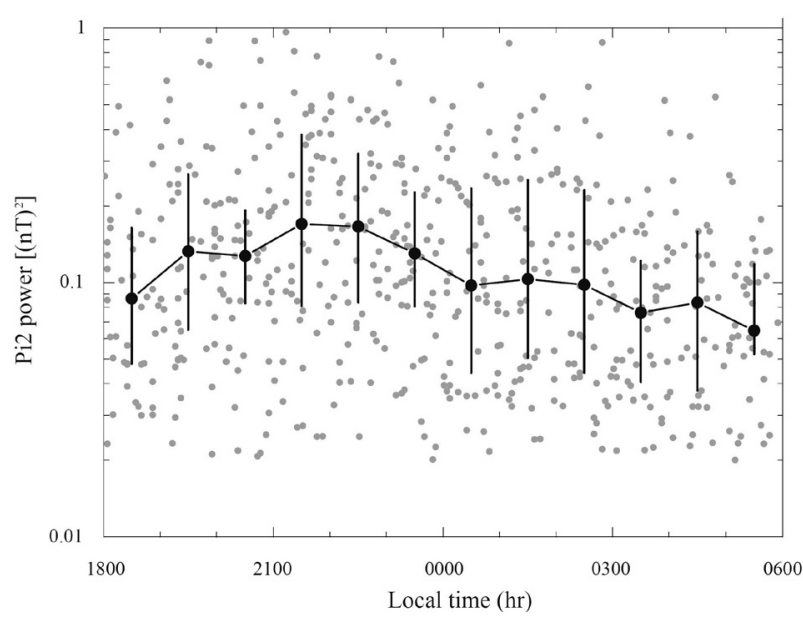

Fig. 5. Power of Pi2 pulsation at Bohyun ground station. The format is the same as in Fig. 3.

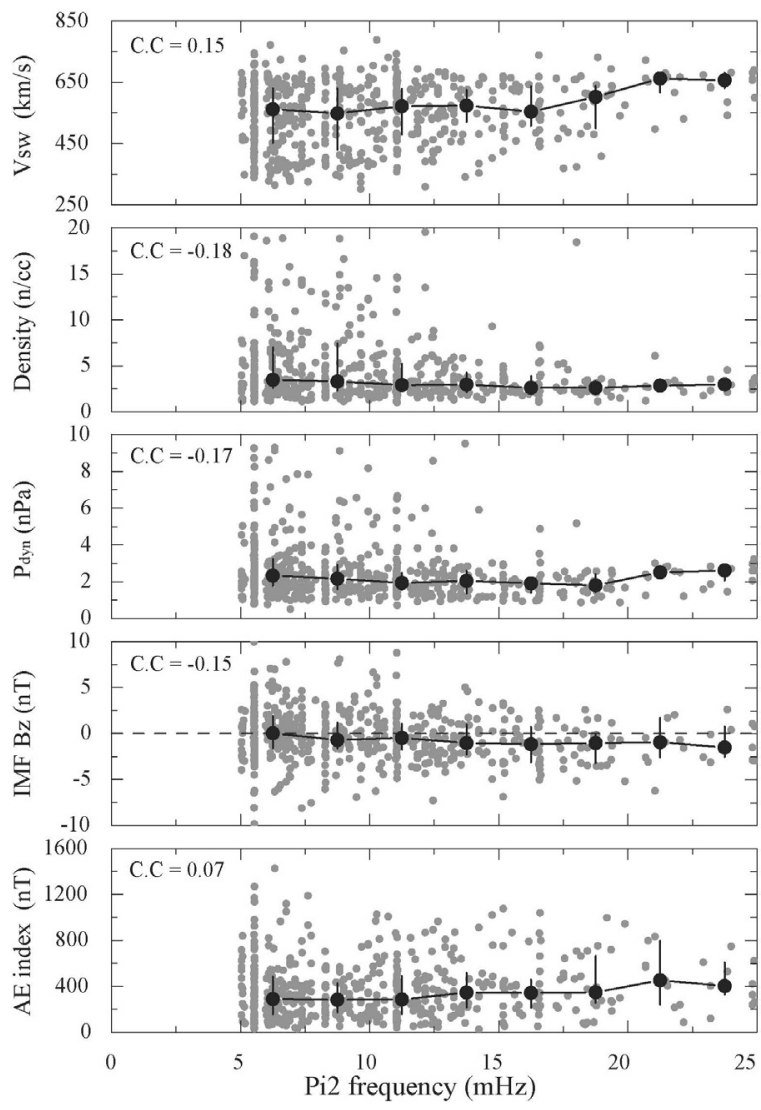

Fig. 6. Dependence of Pi2 pulsation frequency on solar wind condition and $A E$ index. The small gray dots are individual samples, the heavy black dots connected by a line are the medians in $2.5 \mathrm{mHz}$ bins. The vertical error bars connect the lower and upper quartile values. The value of C.C indicates the linear correlation coefficient between the horizontal and vertical value.

highest value at 21 22 LT, and the asymmetry phenomenon of $\mathrm{Pi} 2$ power occurs around the midnight. This result is 


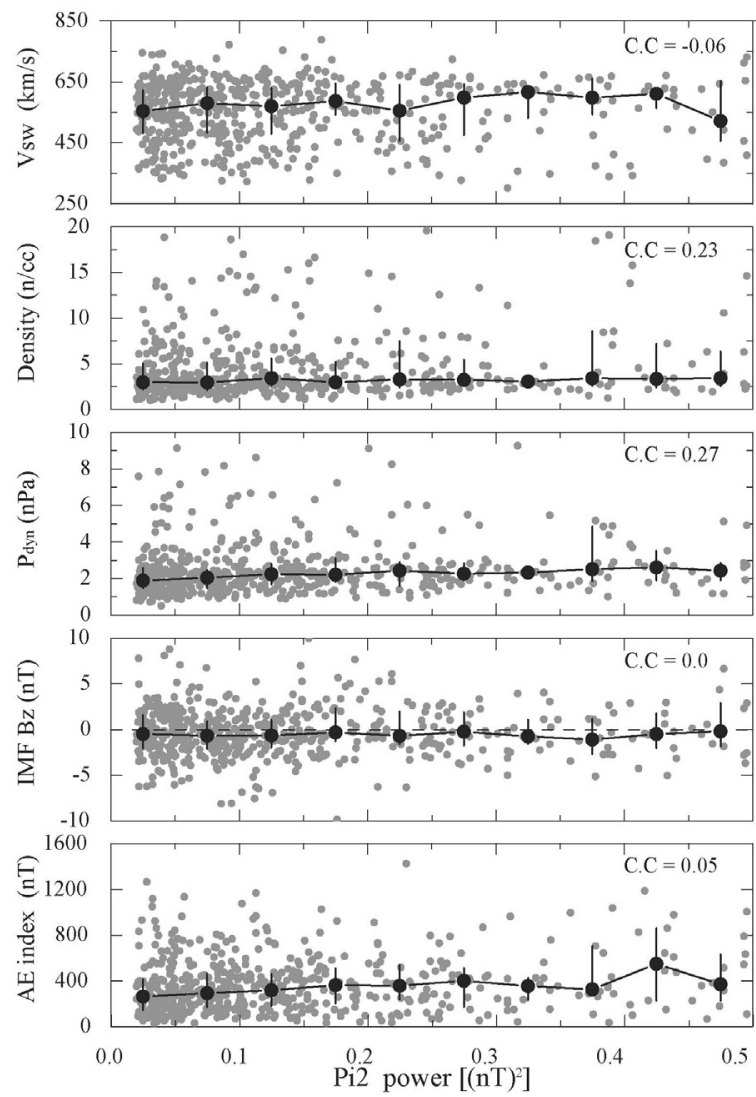

Fig. 7. Dependence of Pi2 pulsation power on solar wind condition and AE index. The small gray dots are individual samples, the heavy black dots connected by a line are the medians into 0.05 (nT) 2 bins. The vertical error bars connect the lower and upper quartile values. The value of C.C indicates the linear correlation coefficient between the horizontal and vertical value.

similar to the result of Takahashi \& Liou (2004).

Fig. 6 shows the relation of $\mathrm{Pi} 2$ pulsation frequency with the solar wind parameters and AE index. In Fig. 6, regarding the speed, density, and dynamic pressure for solar wind and the interplanetary magnetic field Bz-component, the average value data for the 30 minutes which is from 1 hour before to 30 minutes before the Pi2 pulsation occurrence was utilized considering the time it takes for the Earth's magnetosphere to respond to the solar wind parameters (Bargatze et al. 1985). With regard to the AE index, the maximum value for the duration time of $\mathrm{Pi} 2$ pulsation was used. The gray dot represents the value for each Pi2 pulsation frequency, the black dot represents the calculated median value at an interval of $2.5 \mathrm{mHz}$, and the black vertical line connects the low quartile and upper quartile. The correlation coefficient for each data relative to the Pi2 frequency was shown on the upper left side of each figure.

In this data, the solar wind speed and Pi2 frequency had a positive correlation (Troitskaya 1967), but regarding the
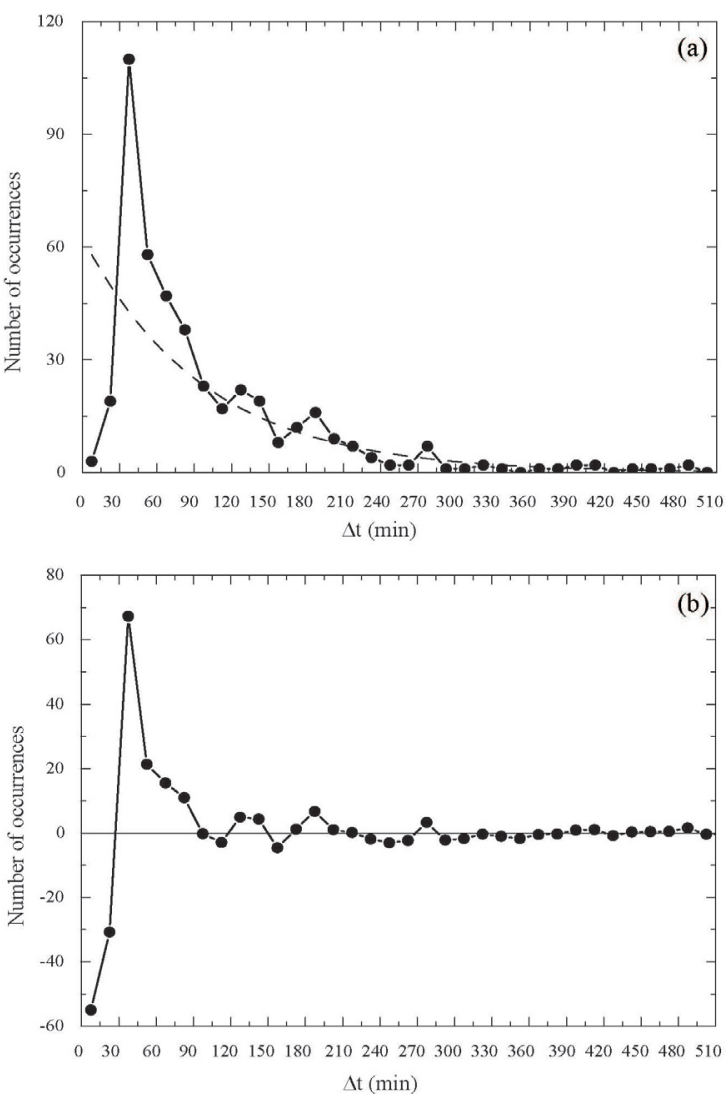

Fig. 8. (a) The recurrent Pi2 pulsation-occurrence Probability function $P$ $(\Delta \mathrm{t})$ obtained by binning the 439 values of $\Delta \mathrm{t}$ into $15 \mathrm{~min}$ bins. The black dashed line indicates Poisson distribution. (b) The result of subtraction the Poisson distribution from the observed distribution $\mathrm{P}(\Delta \mathrm{t})$.

variation of median value, the variation range was small. The solar wind density had a negative correlation, but there seems to be no relation considering the variation of median value. As for the solar wind dynamic pressure, the variation of median value showed similar trend to the solar wind speed at the Pi2 frequency of 20 25 $\mathrm{mHz}$. This is because the solar wind speed is reflected in the calculation of dynamic pressure $\left(\rho \mathrm{V}^{2}: \rho=\right.$ solar wind density, $\mathrm{V}=$ solar wind speed $)$. The interplanetary magnetic field Bz-component had a negative correlation, and it increased toward the south direction depending on the increase of Pi2 pulsation frequency with the variation range of about $1.5 \mathrm{nT}$. As for the AE index, the variation range of median value increased about $120 \mathrm{nT}$ for $7 \sim 22 \mathrm{mHz}$.

Fig. 7 shows the relation of $\mathrm{Pi} 2$ power with the solar wind parameters and $\mathrm{AE}$ index. The correlation coefficient for each data relative to the Pi2 power was shown on the upper right side of each figure. In Fig. 7, according to the correlation coefficient, there was no particular relation between the solar wind parameters and $\mathrm{Pi} 2$ power except 
for the solar wind density and solar wind dynamic pressure. The solar wind density and solar wind dynamic pressure had higher correlation coefficient compared to other solar wind parameters, but regarding the variation of median value, the variation range was insignificant. However, as for the relation to the AE index, it had positive correlation with the $\mathrm{Pi} 2$ power although the correlation coefficient was small, and the variation range of median value increased about $100 \mathrm{nT}$.

Fig. 8 shows the time interval distribution between the consecutive $\mathrm{Pi} 2$ pulsations. In this distribution, the time interval between the two $\mathrm{Pi} 2$ pulsations was investigated, and the time interval of distribution chart was set to 15 minutes. The black dot represents the number of events depending on time interval, and the black dashed line represents the Poisson distribution. The Poisson distribution indicates the theoretical distribution of time interval when the two events occur randomly. The equation for the Poisson distribution is defined as in Eq. (1) (Boas 2006).

$$
P(\Delta T)=439 \times \int_{t 1}^{t 2} \frac{1}{98.33} \exp \left(\frac{\Delta T}{98.33}\right) d \Delta T
$$

where $\Delta T$ is the time interval between the consecutive Pi2 pulsations which occurred between 18:00 and 06:00 LT. The total number of consecutive Pi2 pulsations that occurred in 2008 is 439 , the sum of $\Delta T$ is 719.5 hours, and the average value of $\Delta T$ is 98.33 minutes.

Statistically, in the data from Fig. 8a, there is the interval which does not follow the Poisson distribution. To examine the interval in detail, the Poisson distribution was subtracted from the observed distribution, and the resulting values are shown in Fig. 8b. The first interval is between 37.5 and 82.5 minutes which accounts for about $26 \%$ (115/439) of the total events, and the time interval that has the most events is about 37.5 minutes. The second interval is between 127.5 and 142.5 minutes (2.1 2.4 hours) which accounts for about $2 \%(9 / 439)$ of the total events, and the third interval is between 172.5 and 217.5 minutes (2.9 3.6 hours) which accounts for about $2 \%(9 / 439)$ of the total events.

\section{DISCUSSION}

The number of Pi2 pulsation occurrence was found to be almost evenly distributed in the nightside depending on local time. As for the cause of this study result, the source of Pi2 pulsation occurrence is considered to be near midnight for the Earth's magnetotail region, but when the pulsation occurred, it is thought to be propagated into the broad local time area in the inner magnetosphere for the nightside, which leads to the result.
In this study, the local time dependence of $\mathrm{Pi} 2$ pulsation frequency was observed. This result was consistent with the recent result of Kwon et al. (2012) which used the THEMIS satellite data and low-latitude ground magnetic field station data. Kwon et al. (2012) suggested that the local time dependence of Pi2 frequency is caused by the asymmetry of plasmapause depending on local time, and that this is determined as the Pi2 pulsation period oscillates on the meridional plane.

Other characteristics of low-latitude Pi2 pulsations depending on local time were shown in the Pi2 pulsation power distribution. According to Takahashi \& Liou (2004), the fact that the Pi2 pulsation power depending on local time is higher in the pre-midnight portion is because the highest point of auroral power is around $21 \mathrm{LT}$ and the source region of Pi2 pulsations is located near the eastern head of auroral surge. However, the exact cause is not yet known. In this study, the Pi2 pulsation power was higher in the pre-midnight portion than the post-midnight portion, and the highest Pi2 pulsation power was observed between 21 and 22 LT, which is consistent with the previous study result.

This study examined the relation of Pi2 pulsation characteristics with the solar wind condition and AE index. When the Pi2 pulsation frequency was compared with the solar wind condition and $\mathrm{AE}$ index, there was a correlation that the Pi2 pulsation frequency increases as the speed and dynamic pressure for solar wind and the AE index increase. The interplanetary magnetic field Bz-component had a weak negative correlation. If the $\mathrm{Pi} 2$ pulsations observed had been caused by the plasmaspheric resonance, the major factor that determines the period is the location of plasmapause. The location of plasmapause is inversely proportional to the square root of the electric field (Esw) which is produced by the plasma convection that flows from the magnetotail toward the Earth (Parks 2004). The Esw corresponds to about 10 20\% of solar wind electric field (VBs: V = Vsw, Bs = southward IMF) (Goldstein \& Sandel 2005). Therefore, in this study, the positive correlation between the Pi2 frequency and solar wind speed and the negative correlation between the Pi2 frequency and interplanetary magnetic field Bz-component indicate the migration of plasmapause toward the Earth, which is considered to be related with the increase of Pi2 frequency. The positive correlation with the AE index is considered to be caused by the increase of energy influx to the ionosphere due to the increase of convection in the magnetosphere.

The relation of $\mathrm{Pi} 2$ power with the solar wind parameters and AE index was investigated. For the AE index, the variation range was about $100 \mathrm{nT}$, and the $\mathrm{AE}$ index showed increasing trend as the $\mathrm{Pi} 2$ pulsation power increases. For 


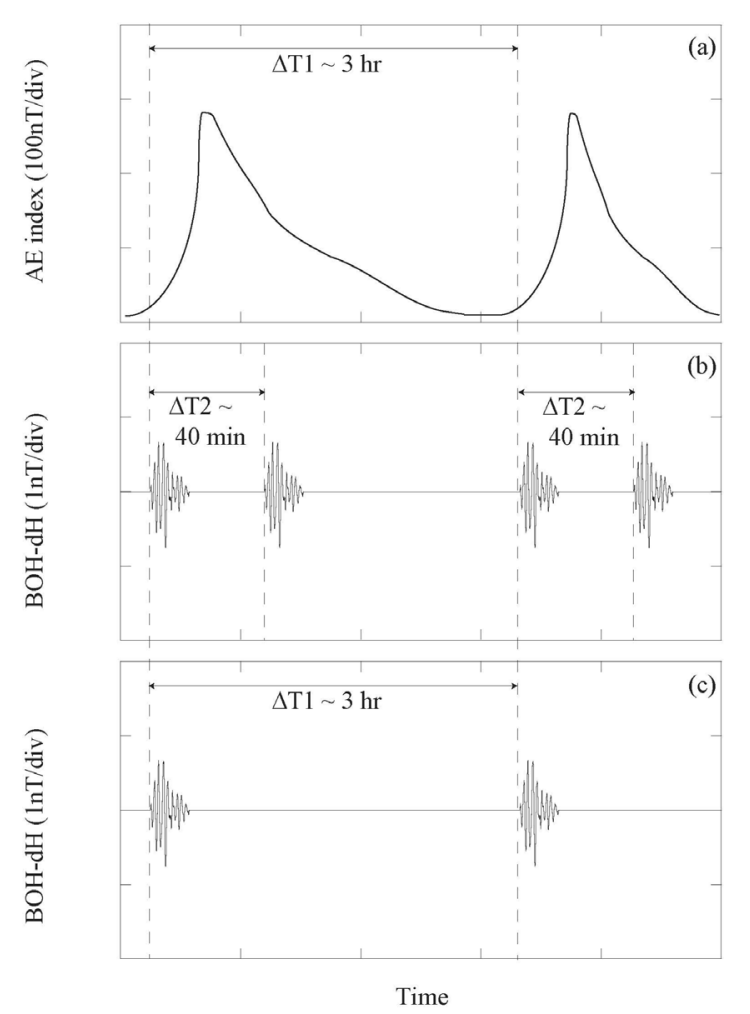

Fig. 9. Schematic illustrations of low-latitude Pi2 pulsations associated with substorm. (a) AL index. (b) Two Pi2 pulsations associated with one substorm. (c) One Pi2 pulsation associated with substorm onset.

the solar wind condition, the variation range was very small, and there was no particular correlation. The relation between the AE index and $\mathrm{Pi} 2$ pulsation power indicates that the stronger Pi2 pulsations occur when the western auroral jet current increases. And the lack of the relation between the Pi2 pulsation power and solar wind condition indicates that the Pi2 pulsation power is not controlled by the external factors, but could be affected by the characteristics within the Earth's magnetosphere.

In this study, the 3 time intervals which could be produced by non-random factors were identified through the statistical analysis of time interval between the consecutive Pi2 pulsations. According to Borovsky et al. (1993), the substorm mostly occurs continuously every 2 to 3 hours. For this study, the time interval between the consecutive Pi2 pulsations was found to be 30 to 40 minutes which is much shorter than the period of substorm, and it accounts for $26 \%$ of the total events. The Pi2 pulsations that have the time interval of 2 to 3 hours were only $4 \%$. Fig. 9 shows the diagram of Pi2 pulsations which occur in association with the substorm. According to the result of this study, for most consecutive $\mathrm{Pi} 2$ pulsations, a number of $\mathrm{Pi} 2$ pulsations occur when a single substorm occurs as shown in Fig. 9b, and the one-to-one correspondence between the $\mathrm{Pi} 2$ pulsation and substorm is very uncommon as shown in Fig. 9c. The 40 minute time interval between the consecutive Pi2 pulsations observed in this study is thought to be the time it takes for the open magnetic lines of force at the tail lobe to move to the center of plasma sheet and to complete the magnetic reconnection after the onset of substorm.

\section{CONCLUSION}

We performed the statistical analysis for total $582 \mathrm{Pi} 2$ pulsations using the low-latitude Bohyun ground magnetic field data $(\mathrm{Kp}>2)$ obtained in 2008.

In this study, the Pi2 pulsations were found to occur evenly depending on nightside local time (18 06 LT). The Pi2 frequency showed increasing trend as it goes from dusk to dawn. This variation of frequency is thought to be caused by the asymmetry of plasmapause depending on local time. In addition, for the Pi2 power distribution based on local time, it showed higher value in the pre-midnight portion than the post-midnight portion, and this is thought to be because the source region of $\mathrm{Pi} 2$ pulsations is located in the pre-midnight portion.

The Pi2 frequency had a positive correlation with the solar wind speed and AE index, and had a negative correlation with the interplanetary magnetic field Bzcomponent. This indicates that the size of plasmapause changed due to the variation of convection caused by the solar wind electric field and thus the Pi2 frequency changed due to the plasmaspheric resonance. The Pi2 power showed no correlation with the solar wind condition, but showed a positive correlation with the AE index. This means that the Pi2 power is determined by the variation in the Earth's magnetosphere rather than the external effects.

As for the time interval distribution between the consecutive $\mathrm{Pi} 2$ pulsations, the intervals that have 30 to 40 minutes time distribution which is shorter than the time interval of general substorm occurrence were observed, and this indicates that a number of $\mathrm{Pi} 2$ pulsations occurred with respect to a single substorm. This time interval is thought to be the time it takes for the open magnetic lines of force at the tail lobe region to move to the center of plasma sheet and to complete the magnetic reconnection.

\section{ACKNOWLEDGMENTS}

The geomagnetic AE and Kp indices was provided by World Data Center C2 (WDC-C2) for Geomagnetism, 
Kyoto University. The Bohyun magnetic field data were provided by the Solar and Space Weather Research Group in Korea Astronomy and Space Science institute. This work was supported by the WCU program through NRF funded by MEST of Korea (R31-10016). The OMNI data were downloaded from the SPDF OMNIWeb service at http:// omniweb.gsfc.nasa.gov/. Work of K.-H. Kim was supported by the Basic Science Research Program through the National Research Foundation of Korea (NRF) funded by the Ministry of Education, Science and Technology (2010-0007393).

\section{REFERENCES}

Bargatze LF, Baker DN, McPherron RL, Hones EW, Magnetospheric impulse response for many levels of geomagnetic activity, JGR, 90, 6,387-6,394 (1985). http://dx.doi.org/10.1029/ JA090iA07p06387

Boas ML, Probability and Statistics, Mathematical Methods in the Physical Sciences, 3rd ed. (Wiley international edition, Hoboken, 2006), 767-770.

Borovsky JE, Nemzek RJ, Belian RD, The occurrence rate of magnetospheric-substorm onsets: Random and periodic substorms, JGR, 98(A3), 3,807-3,813 (1993). http://dx.doi.org/10.1029/92JA02556

Cheng CC, Russell CT, Gao YF, Chi PJ, On consecutive bursts of low-latitude Pi2 pulsations, JASTP, 64, 1,809-1,821 (2002). http://dx.doi.org/10.1016/S1364-6826(02)00190-6

Clauer CR, McPherron RL, Mapping the local time-universal time development of magnetospheric substorms using mid-latitude magnetic observations, JGR, 79, 2,811-2,820 (1974). http://dx.doi.org/10.1029/JA079i019p02811

Goldstein J, Sandel BR, The Global Pattern of Evolution of Plasmaspheric Drainage Plumes, Inner Magnetosphere Interactions, vol. 159, Geophysical Monograph Series, eds. Burch J, Schulz M, Spence H (American Geophysical Union, Washington, D. C, 2005), 1-22.

Kim KH, Kwon HJ, Lee DH, Jin H, Takahashi K, et al., A comparison of THEMIS Pi2 observations near the dawn and dusk sectors in the inner magnetosphere, JGR, 115, A12226 (2010). http://dx.doi.org/10.1029/2010JA016010

Koskinen HEJ, Lopez RE, Pellinen RJ, Pulkkinen TI, Baker DN, et al., Pseudobreakup and substorm growth phase in the ionosphere and magnetosphere, JGR, 98, 5,8015,813 (1993). http://dx.doi.org/10.1029/92JA02482

Kwon HJ, Kim KH, Lee DH, Takahashi K, Angelopoulos V, et al., Local time-dependent Pi2 frequencies confirmed by simultaneous observations from THEMIS probes in the inner magnetosphere and at low-latitude ground stations, JGR, 117, A01206 (2012). http://dx.doi.
org/10.1029/2011JA016815

Nakamura R, Baker DN, Fairfield DH, Mitchell DG, McPherron $\mathrm{RL}$, et al., Plasma flow and magnetic field characteristics near the midtail neutral sheet, JGR, 99, 23,591-23,601 (1994). http://dx.doi.org/10.1029/94JA02082

Nose M, Excitation mechanism of low-latitude Pi2 pulsations: Cavity mode resonance or BBF-driven process?, JGR, 115, A07221 (2010). http://dx.doi.org/10.1029/2009JA015205

Ohtani S, Anderson BJ, Sibeck DG, Newell PT, Zanetti LJ, et al., Multisatellite study of a pseudo-substorm onset in the near-Earth magnetotail, JGR, 98, 19,355-19,367 (1993). http://dx.doi.org/10.1029/93JA01421

Parks GK, Representations of Electric Fields, physics of space plasmas, 2nd eds. (Westview Press, Colorado, 2004), 79-100.

Saito T, Sakurai T, Koyama Y, Mechanism of association between Pi2 pulsation and magnetospheric substorm, J. Atmos. Terr. Phys, 38, 1,265-1,277 (1976). http://dx.doi. org/10.1016/0021-9169(76)90135-5

Takahashi K, Liou K, Longitudinal structure of low-latitude Pi2 pulsations and its dependence on aurora, JGR, 109, A12206 (2004). http://dx.doi.org/10.1029/2004JA010580

Takahashi K, Ohtani S, Anderson BJ, Statistical analysis of Pi2 pulsations observed by the AMPTE CCE spacecraft in the inner magnetosphere, JGR, 100(A11), 21,92921,941 (1995). http://dx.doi.org/10.1029/95JA01849

Troitskaya VA, Micropulsations and the State of the Magnetosphere, Solar-Terrestrial Physics, eds. King JW \& Newman WS (Academic Press, London, 1967), 213-274. 